\title{
Straddle injuries to the bulbar urethra: management and outcome in 53 patients
}

Elgammal MA

Department of Urology, Assiut University Hospital, Assiut, Egypt

Int Braz J Urol. 2009; 35: 450-8

Objective: To describe our experience with blunt injuries to the bulbar urethra and their late sequelae to identify factors that may affect patient outcome.

Materials And Methods: A retrospective study was performed on 53 male patients who presented, between January 2001 and December 2005, with blunt traumatic injury to the bulbar urethra. The definitive diagnosis of urethral rupture was made by retrograde urethrography, where urethral rupture was classified into partial or complete. The minimum follow-up period was 3 years. The initial management was either suprapubic cystostomy or endoscopic urethral realignment over a urethral catheter using a cystoscope to pass a guide-wire over which the catheter was inserted. Stricture formation was managed by visual internal urethrotomy (VIU) for passable strictures and urethroplasty (stricture excision and re-anastomosis) for impassable strictures or recurrence after VIU. The follow-up period was three years. The results were analyzed by SPSS software (chisquare and Student's-t-test).

Results: Stricture formation occurred in 19 of 22 patients $(86 \%)$ with complete urethral rupture and in 10 of $31(32 \%)$ with partial rupture $(\mathrm{p}<0.001)$. Strictures occurred in 11 of $31(35 \%)$ patients treated initially with suprapubic cystostomy and in 18 of $22(82 \%)$ treated with primary urethral realignment $(\mathrm{p}<0.001)$. The success rate after VIU was 15\% (4 of 26 patients) and after urethroplasty it was $96 \%$ (24 of 25 patients) $(\mathrm{p}<0.001)$.

Conclusions: Suprapubic cystostomy is better than urethral realignment and catheterization as primary management after straddle injury to the bulbar urethra. Stricture excision and re-anastomosis is better than VIU as delayed management for strictures that develop after straddle injury to the bulbar urethra.

\section{Editorial Comment}

While a few of the above articles are old, they illustrate important teaching points about how urethral injury etiology dictates outcome and the best choice for management.

Blunt crush injuries to the urethra typically results in a short segment of spongiofibrosis that occurs in the mid bulbar urethra. Stricture etiology, location and length typically dictate the type of repair selected and the success of the long term outcome. With a blunt injury, the stricture is typically less than $2 \mathrm{~cm}$ and the natural elasticity of the mobilized urethra can bridge the gap. The spongiofibrosis from a straddle injury is isolated to a short segment, while the rest of the urethra and the rest of the corpus spongiosum are normal. Inflammatory strictures typically cause a more diffuse spongiofibrosis, and thus are often best managed by an onlay skin flap or buccal mucosal graft. 
Straddle injuries are not to be confused with the stenoses that occur from pelvic fracture. With pelvic fracture, the injury is a distraction injury where there is disruption of the urethra and corpus spongiosum at the level of the membranous - bulbar junction or the membranous and the prostate. Here there is no real spongiosum fibrosis and "urethral stricture" - but scar tissue that fills the gap. Primary realignment is the preferred management of such injuries because it a distraction injury and not a stricture. Historically, the outcomes of primary realignment are a reduction in urethral stricture by $50 \%$, while the rates of erectile dysfunction and incontinence are the same as a suprapubic tube. Furthermore, the eventual stricture that does occur is often shorter and more amenable to urethrotomy.

From the above abstracts, I think the conclusion that straddle injuries should be managed by suprapubic tube alone, as the best management that should be followed. Intuitively, we would assume that the Denis Browne principle would apply here and stenting would promote epithelialization. However, until a randomized prospective trial takes pace - and I doubt that any such study will be done soon - we should resist the temptation to primarily realign the urethra. As to urethral penetrating urethral injuries from low velocity gunshot wounds (no delayed ischemia or blast effect) the site of injury is typically short. A short area of injury can be bridged by adequate mobilization and natural elasticity of the urethra, particularly in the bulbar urethra. In the penile urethra, over mobilization and an anastomosis on tension may result in chordee or stricture failure. Primary realignment of a short penile urethral injury is not the first treatment of choice - but rather surgical exploration and primary repair. When the defect is too long (more than $1 \mathrm{~cm}$ or so), urethral marsupialization and a two stage repair (in the method of Johansson) is probable best.

Dr. Steven B. Brandes Associate Professor, Division of Urologic Surgery Washington University in St. Louis

St. Louis, Missouri, USA

E-mail: brandess@wudosis.wustl.edu

\section{PATHOLOGY}

\section{Does perineural invasion on prostate biopsy predict adverse prostatectomy outcomes?}

Loeb S, Epstein JI, Humphreys EB, Walsh PC

James Buchanan Brady Urological Institute and the Department of Urology, Johns Hopkins Medical Institutions, Baltimore, MD, USA

BJU Int. 2009 Aug 19. [Epub ahead of print]

Objective: To determine the relationship between perineural invasion (PNI) on prostate biopsy and radical prostatectomy (RP) outcomes in a contemporary RP series, as there is conflicting evidence on the prognostic significance of PNI in prostate needle biopsy specimens. 